\title{
Review
}

\section{Registration methods in radiotherapy}

\author{
Paweł Czajkowski ${ }^{a}$, Tomasz Piotrowski ${ }^{b, c, *}$ \\ a Department of Medical Physics, Gdynia Oncology Centre, Gdynia, Poland \\ b Department of Electroradiology, Poznan University of Medical Sciences, Poznan, Poland \\ c Department of Medical Physics, Greater Poland Cancer Centre, Poznan, Poland
}

\section{A R T I C L E I N F O}

\section{Article history:}

Received 28 June 2018

Accepted 6 September 2018

Available online 10 October 2018

\section{Keywords:}

Image registration

Radiotherapy

Rigid registration

Deformable registration

\begin{abstract}
A B S T R A C T
Purpose: The aim of this study is to present a short and comprehensive review of the methods of medical image registration, their conditions and applications in radiotherapy. A particular focus was placed on the methods of deformable image registration.

Methods: To structure and deepen the knowledge on medical image registration in radiotherapy, a medical literature analysis was made using the Google Scholar browser and the medical database of the PubMed library.

Results: Chronological review of image registration methods in radiotherapy based on 34 selected articles. A particular attention was given to show: (i) potential regions of the application of different methods of registration, (ii) mathematical basis of the deformable methods and (iii) the methods of quality control for the registration process.

Conclusions: The primary aim of the medical image registration process is to connect the contents of images. What we want to achieve is a complementary or extended knowledge that can be used for more precise localisation of pathogenic lesions and continuous improvement of patient treatment. Therefore, the choice of imaging mode is dependent on the type of clinical study. It is impossible to visualise all anatomical details or functional changes using a single modality machine. Therefore, fusion of various modality images is of great clinical relevance. A natural problem in analysing the fusion of medical images is geographical errors related to displacement. The registered images are performed not at the same time and, very often, at different respiratory phases.
\end{abstract}

○ 2018 Greater Poland Cancer Centre. Published by Elsevier Sp. z o.o. All rights reserved.

\section{Introduction}

The development of computer technologies in the management of medical images achieved through diagnostic X-ray tests, computer tomography (CT), magnetic resonance (MR) or positron emission tomography (PET), has enabled those images to be compared directly on a computer screen. One of the methods for advanced comparison of medical images involves the alignment of the images followed by assessment of visible structures of the internal anatomy of patient's body. The ability to overlay images with different modalities, e.g. CT and MR, which complement the information of patient's anatomy by utilising different physical phenomena in the

\footnotetext{
* Corresponding author at: Department of Medical Physics, Greater Poland Cancer Centre, Garbary 15, $61-866$ Poznan, Poland.

E-mail address: tomasz.piotrowski@me.com (T. Piotrowski).

https://doi.org/10.1016/j.rpor.2018.09.004

1507-1367/@ 2018 Greater Poland Cancer Centre. Published by Elsevier Sp. z o.o. All rights reserved.
} 
process of being generated, has considerably improved the precision of diagnostic and clinical decisions. The process of overlaying medical images is referred to as 'registration', while the effect of the registration is known as 'fusion'.

The tools for medical image registration are constantly improved. Almost every year, new technological solutions are developed (computer architecture - hardware) to shorten the time and enhance the precision of registration (mathematical algorithms used during registration - software). The accuracy of medical image fusion is very significant for a number of medical disciplines, including radiotherapy. In the case of radiotherapy, a performed and verified image fusion with the lowest geographical error enables a clear segmentation of the planning target volume (PTV) and the organs at risk (OAR) and, consequently, the creation of a treatment plan. ${ }^{1-3}$ Registration of medical images and the evaluation of the fusion obtained is also one of the main methods to control the delivery of a treatment plan on a therapeutic machine, i.e. during patient irradiation. Irradiation during which a regular image-based control is performed is referred to as image guided radiation therapy (IGRT)..$^{4-7}$

A number of review studies have been published on image registration. But they are of a general nature and relate to various fields of science, from image theory through technical application to medical sciences. The aim of this particular study is to review the methods of medical image registration, their conditions and applications in radiotherapy. A particular focus was placed on the methods of deformable image registration.

\section{Material and method}

To structure and deepen the knowledge on medical image registration in radiotherapy, a medical literature analysis was made using the Google Scholar browser and the medical database of the PubMed library. The key words used in the search were image registration in radiotherapy, deformable image registration in radiotherapy, adaptive radiotherapy. Out of dozens of reports on medical image registration, we only selected those concerned with particular modes of registration and their use in radiotherapy. A particular attention was given to the papers describing the use of demon and B-spline deformable registration types.

\subsection{Image registration - process}

Medical image registration may be divided according to a number of factors. ${ }^{8-10}$ Therefore, to perform the registration, some landmarks must first be identified for the input image to be aligned to the reference image. Usually, these are characteristic elements of patient's anatomy that are visible in both images, such as selected bony structures or clear-cut and distinctly visible blocks of soft tissue. Currently, there is a variety of algorithms to be used for the purpose of medical image registration. ${ }^{11}$ The choice of an algorithm will determine the method and type of transformation the input image will undergo when being registered with the reference. The final and the key part of the whole process is the evaluation of the fusion received. The evaluation should take into account both the algorithm-specific quantitative parameters and the qualitative parameters that specify diagnostic utility of the registration or, in other words, assess if the registration has delivered some added information on patient's anatomy as compared to that provided by the reference image alone.

\subsection{Chronological review of image registration methods in radiotherapy}

The first medical image registration methods implemented in computer programmes and used in radiotherapy were those based on simple and complex rigid transformation algorithms. They rely on mathematical transformations, such as rotation and translation along each axis of the Cartesian coordinate system, as well as scaling and shearing of an image projected onto a reference. ${ }^{12-14}$ Simple rigid registration uses the first two of the above transformations, while complex rigid transformation also involves scaling and shearing. Fig. 1 shows a schematic of the basic transformations used in rigid image registration methods.

In rigid registration, transformations are performed in a 3D space and images are matched according to the properties of pixels or voxels or by alignment of contours associated with selected anatomical structures as shown in the images. Rigid registration is global in nature, meaning that particular parts of the input image are not able to be deformed in relation to one another, and the fusion obtained is a superposition between the input and reference image. Superposition of images is, in turn, conditioned upon the underlying registration parameters (points, surfaces or mapping volumes that are identical and clearly specified in both images being compared). ${ }^{14,15}$

Rigid registration is an indispensable tool in patient positioning before the delivery of fractional dose. In that case, rigid registration of $\mathrm{CT}$ images that formed the basis for treatment plan with CT images (e.g. cone beam computed tomography $\mathrm{CBCT}$ ) allows to determine the values by which the therapeutic table with the patient on it should be moved so that the current patient's position reflects as far as possible the position taken while the CT images used in treatment plan were being made.

Unfortunately, the rigid registration methods may not be reliable in determining the target volume and organs at risk in the course of treatment planning. Registering MR or PET images with reference CT images should provide measurable benefits of added information on patient's anatomy. However, the global nature of rigid registrations often leads to unacceptable discrepancies between the positions of particular anatomical structures in images being compared. The discrepancies arise from the fact that images can be taken at different times or for different body positions.

In the assessment of anatomical changes that occur in the patient's body during radiation therapy, rigid registration methods enable a qualitative visualisation of discrepancies between reference CT images (treatment planning, images generated before the onset of therapy) and the input CBCT images generated during radiotherapy. Nevertheless, rigid transformation parameters (rotation, shift, scaling) do not make it possible to quantify those changes. Nor is it possible, based on those parameters, to map and deform the 


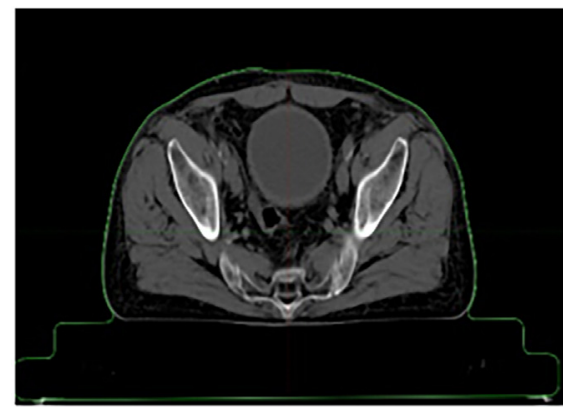

original image

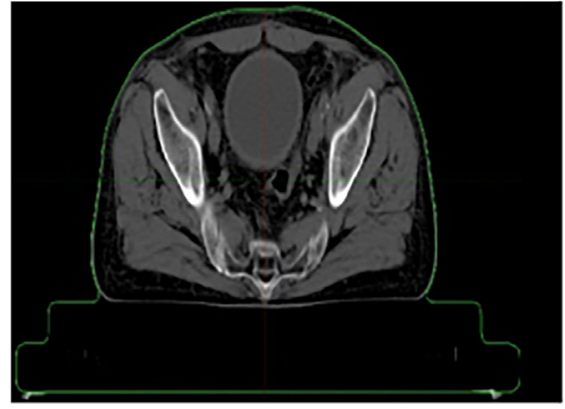

resized image

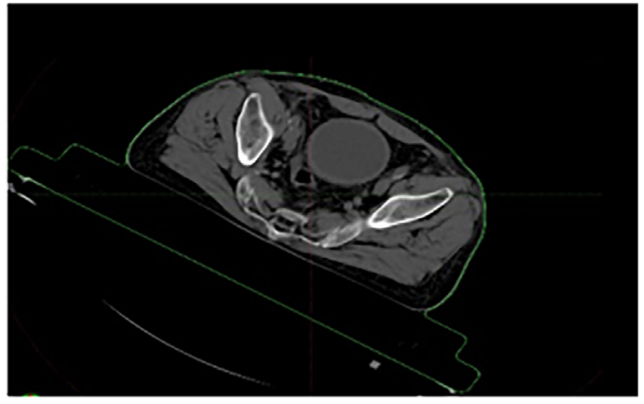

rotated image

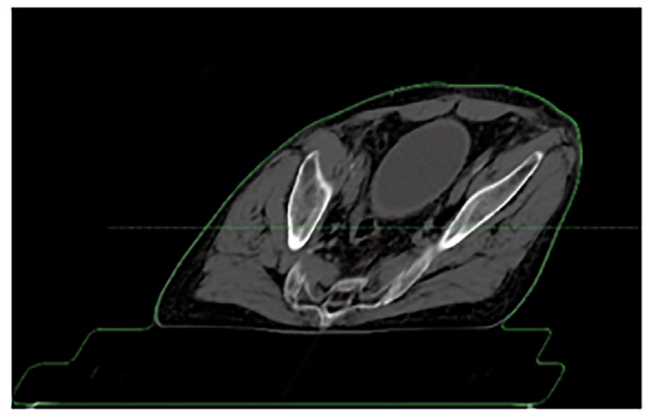

shearing image

Fig. 1 - Examples of transformations applied in rigid image registration (own data).

dose deposited during irradiation in a non-linearly changing anatomy of the patient's body.

Some hope for solution of the issues related to the rigid registration methods was offered by the registration based on projective transformation algorithms employing the process of perspective scaling. Perspective scaling allows the re-scaling of particular parts of an input image by various nonidentical rates. Thus, it is possible to enlarge a selected part of an input image in relation to the other parts. ${ }^{12,14}$ Projective registrations are both global (matching the whole input image with the reference) and local (matching a selected part of the input image with the reference) in nature. Unfortunately, scaling and shifts of particular parts of the image during projective registration are linear in nature as in the case of rigid registration. Hence, just like rigid registrations, they are likely to produce a substantial misalignment. ${ }^{15}$ Therefore, a need has arisen for methods using fully elastic transformations which, apart from re-scaling particular image elements, would enable them to be non-linearly shifted in relation to one other. Such transformations are referred to as deformable image registration (DIR), whereas registration based on such transformations is known as deformable registration.

Currently, the most common deformable registrations commercially available in computer software used in radiotherapy are those employing algorithms based on elastic transformations that involve the analysis of voxel intensity in the referred image as compared to the reference image (demon registration) and on the analysis of B-spline functions. ${ }^{16,17}$

\subsection{Demon deformable registration}

The concept of a non-linear image registration using voxel intensity of images being matched is based on the thermodynamic paradox proposed by James Clerk Maxwell in 1867 (Fig. 2).

It assumes that a mixture of gases composed of molecules $a$ and $b$ contains an intrinsic semi-permeable membrane capable of 'differentiating' between the molecules. The membrane only allows molecules $a$ to diffuse in the direction $\mathrm{A}$, and molecules $b$ to diffuse in the direction $B$. To account for the decreased entropy of the system, which is contrary to the second law of thermodynamics, Maxwell proposed in his thought experiment the existence of a being who 'could' differentiate between molecules - a demon. In fact, in 2013, Maxwell's demon was theoretically proved to exist as a quantum dot.

That concept has been used in the demon-type algorithms that are examples of image registration based on diffusive models of image deformation. They assume that appropriate image voxels are properly assigned by means of a force field. According to the level of saturation, each target image voxel is assigned a reference image voxel by means of a field based on optical flow. ${ }^{18}$ It is assumed that the reference image is a rigid image whose external contour is a membrane where Maxwell's demons are located. The demons are of a local nature and are responsible for pushing a voxel into the reference image if it is labelled 'in' or pushing it out of the reference image if it is labelled 'out' (Fig. 3). The level of deformation of the input image during a demon-type registration is based on a 


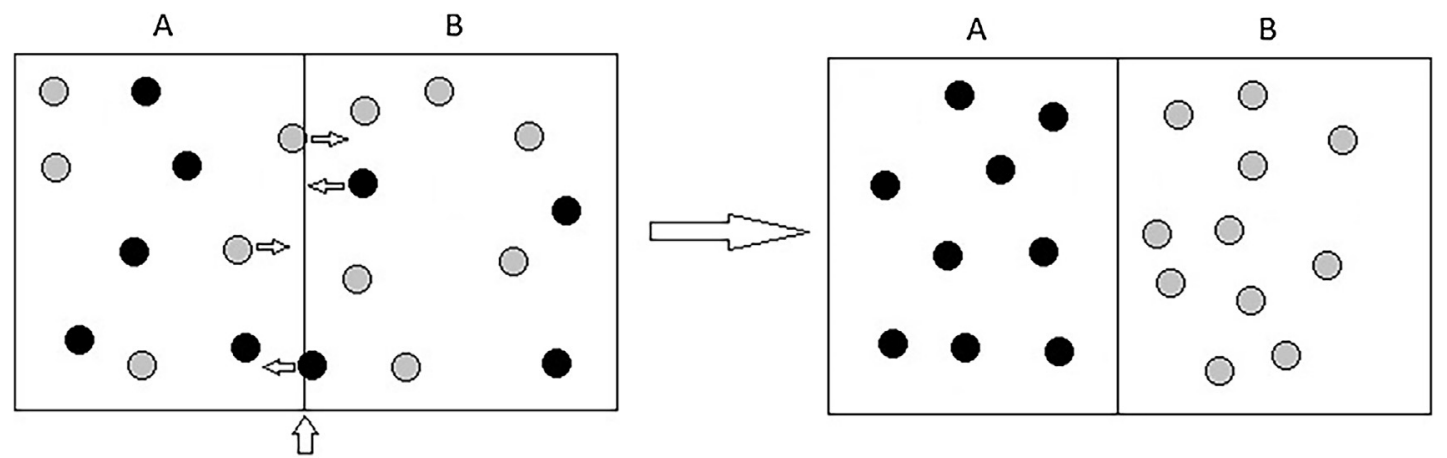

membrane with Maxwell's Demons

Fig. 2 - Gas mixture and Maxwell's demons.

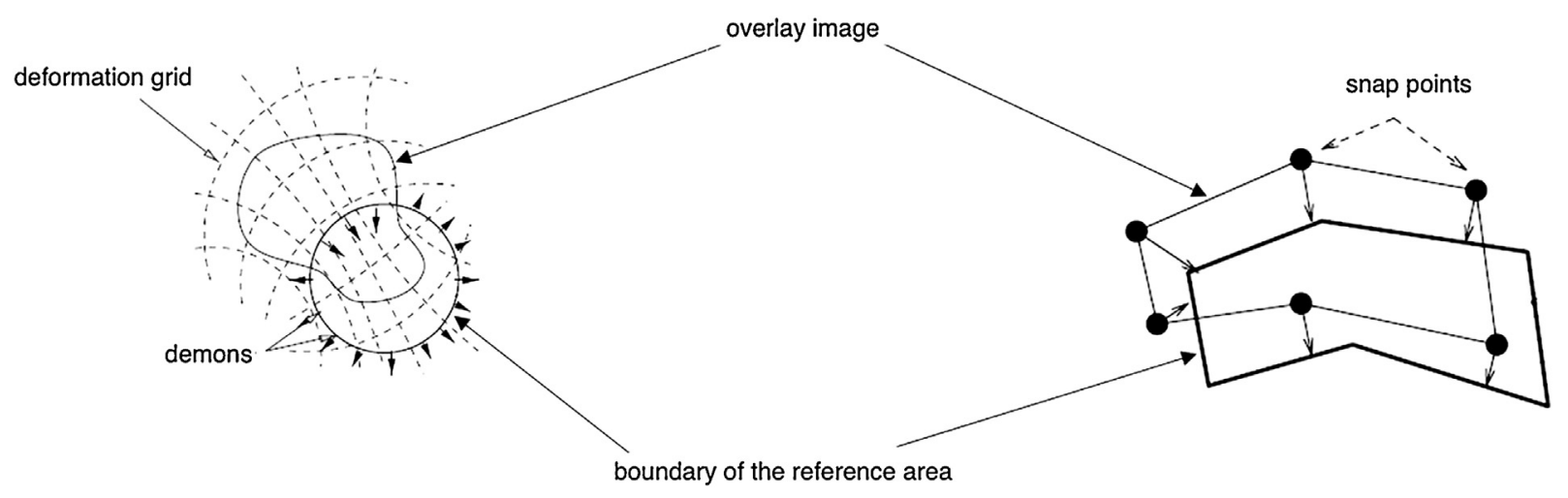

Fig. 3 - Diffusive model of image registration.

vector field of the movement between the two images - optical flow.

The main condition for optical flow is that both images remain bright. In a two-dimensional space, brightness depends on the point location $(x, y)$ and time $(t)$ and is defined as:

$I=I(x, y, t)$

Considering that brightness is invariable in time and extending Eq. (1) to Taylor series, the following formula can be proposed:

$I_{x} u+I_{y} v+I_{t}=0$, where $: I_{x}=\partial I / \partial x, I_{y}=\partial I / \partial y, u=d x / d t$,

$v=d y / d t$ and $I_{t}=\partial I / \partial t$

Eq. (2) is the condition for optical flow. There are three main groups of solutions for this linear equation based on correlative, frequency and gradient. ${ }^{19}$ The gradient-based solutions rely on spatial derivatives of image intensity and are implemented, among others, in commercially available solutions, such as Smart Adaptive and Smart Segmentation in the Ecplipse $^{\mathrm{TM}}$ (Varian Medical Systems, Palo Alto, USA) radiotherapy planning system, MIM Software (MIM Software Inc., Cleveland, USA) therapeutic data management software, and Accuray Precision ${ }^{\mathrm{TM}}$ (Accuray Inc, Madison, USA) radiotherapy planning system.

\subsection{B-spline deformable registration}

The main objective of registering medical images is to find a geometrical transformation $\mathrm{T}$ to transform as accurately as possible the image sequence $B$, usually with different modalities, into the image sequence A (Fig. 4).

In the case of demon-based deformable transformation algorithms, such a transformation results from the solution of the equation that constitutes the condition for optical flow. In contrast to demons, B-spline algorithms rely on the description of transformation $\mathrm{T}$ based on the analysis of the spline functions that connect voxels in space involving a set of control points. ${ }^{20}$ The image sequence $B$ is subjected to deformation by using spline functions that have become a tool to design any forms made of continuous curved lines.

To model of contour, sometimes very complicated, a mathematical method of fitting polynomial curve segments is used and represented as a B-spline. B-spline functions are a particular case of spline functions - standardised by base functions. The domain of those functions falls into the range of $t \in[0,1]$, while the $m+1$ sequence of values $u_{i}$ divides that range into sub-ranges where particular curves are defined. Values $u_{i}$ are called B-spline nodes. Any point of the curve is expressed by the equation:

$v(x)=\sum_{i} p_{i} \beta_{i}(x)$ 


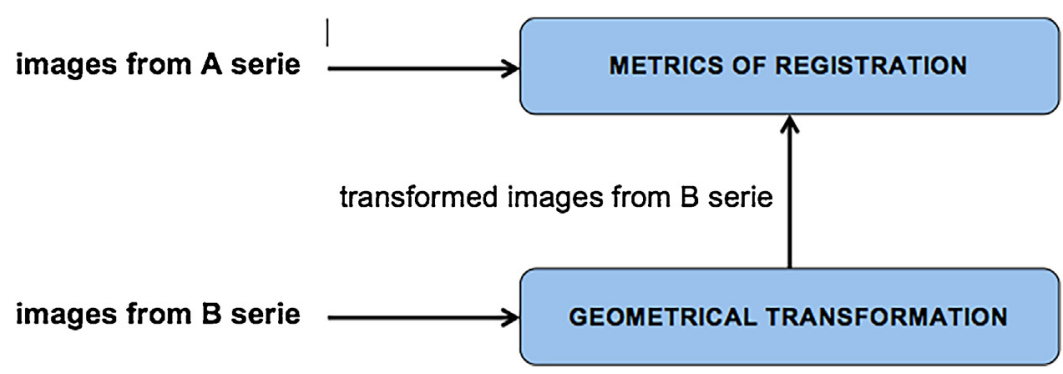

Fig. 4 - Schematic image registration process.

where:

$p_{i}-$ control points, scaling vectors

$\beta_{i}(\mathrm{x})$ - standardised B-spline functions, typically represented

3rd degree polynomials

To interpolate a contour using B-spline functions, the interpolating polynomial needs to be calculated for each section between two successive nodes. The coefficients of those polynomials are set in such a way as to ensure continuity of the function and its derivative to $1-n$ degree, assuming that $n$ is the degree of the polynomial.

The function fitting technique consists in user's choosing control points (nodes). Usually, these are characteristic points of a medical image: anatomical changes, bony structures. Another benefit of B-spline algorithms is that the method for obtaining deformations depends entirely on computer processing power. The most frequent graphic representation of B-spline deformable registration is the exposure of curve grid deformation and the consequent deformable warp map
(Fig. 5). The B-spline registration technology is implemented in the Velocity ${ }^{\mathrm{TM}}$ imaging data management system (Varian Medical Systems, Palo Alto, USA).

\subsection{Deformable registration quality control}

Deformable registration quality control and validation methods are described in Report 132 of the American Association of Physicists in Medicine. ${ }^{21}$ It includes recommendations and detailed information on actions to be taken when implementing a medical image registration system. Before introducing the algorithm to clinical use, an obligatory quantitative and qualitative inspection of the medical image registration process has to be performed. A quality control needs also to be done for routine clinical trials. The report contains detailed recommendations on what structures should be compared in specific clinical areas and how to visualise the fusion of images to prevent as many errors as possible. The 132 AAPM Working Group recommends in their report to use the

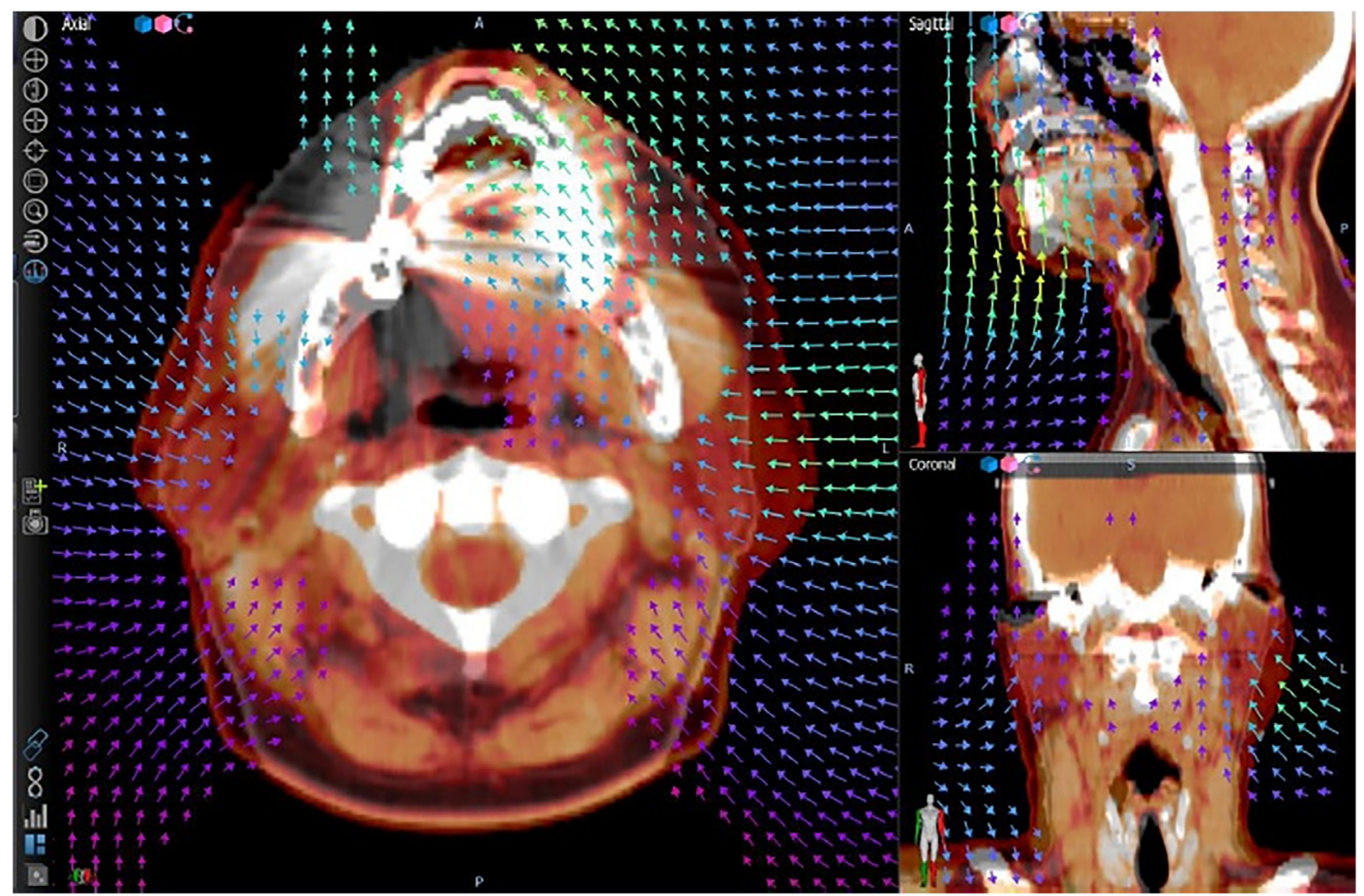

Fig. 5 - Deformable warp map (own data). 


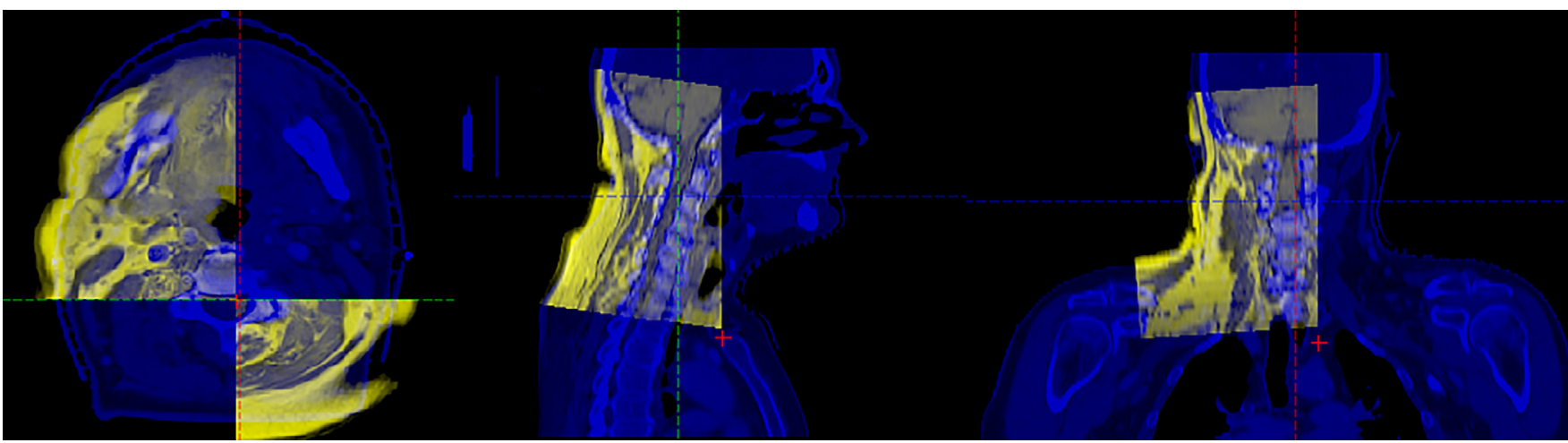

Fig. 6 - Medical image fusion.

so-called chessboard (Fig. 6) to evaluate the fusion of medical images as a qualitative tool.

To perform a quantitative validation of the algorithm, a socalled target registration error (TRE) needs to be determined, which is far from easy in the case of multi-modal volumes. It is a mean of differences between the same points of the referred and reference image during the registration process. For ideally aligned images, TRE is 0 . Another way to carry out a quantitative quality control for the algorithms is to determine the mean distance to agreement with tolerance of $2-3 \mathrm{~mm} .{ }^{22,23}$ The complement way of evaluation is based on the comparisons of the real or planned motions that are simulated in physical or computational phantoms with the results of registrations. ${ }^{24-29}$

Despite the commercial implementation, scientists are constantly developing new methods of registration, contributing constantly to their precision and efficiency. ${ }^{30-32}$ It is important because current practice shows that there is no ideal registration algorithm and the selection of one of them should be dictated by specific clinical situations. ${ }^{33,34}$

\section{Conclusion}

The primary aim of the medical image registration process is to connect the contents of images. What we want to achieve is a complementary or extended knowledge that can be used for more precise localisation of pathogenic lesions and continuous improvement of patient treatment. Therefore, the choice of an imaging mode is dependent on the type of clinical study. It is impossible to visualise all anatomical details or functional changes using a single modality machine. Therefore, fusion of various modality images is of great clinical relevance. A natural problem in analysing the fusion of medical images is geographical errors related to displacement. The registered images are performed not at the same time and, very often, at different respiratory phases.

\section{Conflict of interest}

None declared.

\section{Financial disclosure}

None declared.

\section{REFERENCES}

1. Piotrowski T, Ryczkowski A, Adamczyk M, Jodda A. Estimation of the planning organ at risk volume for the lenses during radiation therapy for nasal cavity and paranasal sinus cancer. J Med Imaging Radiat Oncol 2015;59:743-50.

2. Yang J, Beadle BM, Garden AS, Schwartz DL, Aristophanous M. A multimodality segmentation framework for automatic target delineation in head and neck radiotherapy. Med Phys 2015;42:5310-20.

3. Piotrowski T, Kazmierska J, Sokołowski A, et al. Impact of the spinal cord position uncertainty on the dose received during head and neck helical tomotherapy. J Med Imaging Radiat Oncol 2013;57:503-11.

4. Zitova B, Flusser J. Image registration methods: a survey. Image Vis Comput 2003;21:997-1000.

5. Adamczyk M, Piotrowski T, Adamiak E, Malicki J. Dosimetric consequences of prostate-based couch shifts on the precision of dose delivery during simultaneous IMRT irradiation of the prostate, seminal vesicles and pelvic lymph nodes. Phys Med 2014;30:228-33.

6. Schultze J, Both M, Luetzen U. Zagadnienie obrazowania w radioterapii ze szczególnym uwzględnieniem brachyterapii. Nowotwory 2007;57:376-82.

7. Piotrowski T, Kaczmarek K, Bajon T, Ryczkowski A, Jodda A, Kaźmierska J. Evaluation of image-guidance strategies for prostate cancer. Technol Cancer Res Treat 2014;13:583-91.

8. Sindhu Madhuri G. Classification of image registration techniques and algorithms in digital image processing - a research survey. Int J Comput Trends Technol 2014;15:78-82.

9. Van Den Elsen PA, Pol EJD, Vierger MA. Medical image matching: a review with classification. IEEE Eng Med Biol 1993;12:26-39

10. Brown Gottesfeld L. A survey of image registration techniques. ACM Comput Surv 1992;24:325-76.

11. Pluim JP, Maintz JB, Viergever MA. Mutual information based registration of medical images: a survey. IEEE Trans Med Imaging 2003;22:986-1004

12. Ashburner J, Friston KJ. Rigid body registration. In: Human brain function. 2nd ed. San Diego: Academic Press, Elsevier Science; 2004

13. Gupta S, Chakarvarti SK, Zaheerudin. Image registration methods: a short review. Am J Algorithms Comput 2013;1:39-49. 
14. Gupta N, Garg NK. Review on different techniques of image registration. Int J Eng Comput Science 2014;3:5093-7.

15. Wyawahare MV, Patil PM, Abhyankar HK. Image registration techniques: an overview. Int J Signal Process 2009;2:11-28.

16. Piotrowski T, Ryczkowski A, Kazmierska J. B-spline registration based on new concept of an intelligent masking procedure and GPU computations for the head and neck adaptive tomotherapy. Technol Cancer Res Treat 2012;11:257-66.

17. Pennec X, Cachier P, Ayache N. Understanding the "demon's algorithm": 3D non-rigid registration by gradient descent. In: Taylor C, Colchester A, editors. Medical image computing and computer-assisted intervention - MICCAI'99. MICCAI 1999. Lecture notes in computer science, vol. 1679. Berlin, Heidelberg: Springer; 1999.

18. Skalski A, Zieliński T. Segmentacja i dopasowanie cyfrowych obrazów medycznych: przetwarzanie nagrań wideo-endoskopowych strun głosowych oraz danych tomograficznych zmian rakowych. Pomiary Automatyka Kontrola 2008;54:330-3.

19. Thirion JP. Image matching as a diffusion process: an analogy with Maxwell's demons. Med Image Anal 1998;2:243-60.

20. Kessler M. Jak podnieść precyzję radioterapii: obrazowanie skojarzone i czterowymiarowe (4D). Nowotwory 2008;58:493-8.

21. Brock KK, Mutic S, McNutt TR, Li H, Kessler ML. Use of image registration and fusion algorithms and techniques in radiotherapy: report of the AAPM Radiation Therapy Committee Task Group No. 132. Med Phys 2017;44(7):e43-76.

22. Jamema SV, Phurailatpam R, Paul SN, Joshi K, Deshpande DD. Commissioning and validation of commercial deformable image registration software for adaptive contouring. Phys Med 2018;47:1-8.

23. García-Mollá R, de Marco-Blancas N, Bonaque J, Vidueira L, López-Tarjuelo J, Perez-Calatayud J. Validation of a deformable image registration produced by a commercial treatment planning system in head and neck. Phys Med 2015;31:219-23.

24. Miyakawa S, Tachibana H, Moriya S, Kurosawa T, Nishio T. Evaluation of deformation parameters for deformable image registration-based ventilation imaging using an air-ventilating non-rigid phantom. Phys Med 2018;50: 20-5.

25. Kanakavelu N, Samuel EJJ. Accuracy in automatic image registration between MV cone beam computed tomography and planning $\mathrm{kV}$ computed tomography in image guided radiotherapy. Rep Pract Oncol Radiother 2016;21(5):487-94.

26. Rafic KM, Amalan S, Peace BST, Ravindran BP. Extended localization and adaptive dose calculation using HU corrected cone beam CT: Phantom study. Rep Pract Oncol Radiother 2018;23(2):126-35.

27. Skett $\mathrm{S}$. Testing of deformable image registration in radiotherapy using computational phantoms. Phys Med 2014;30:e32-3.

28. Kanakavelu N, Ravindran AM, Samuel EJJ. Evaluation of mechanical and geometric accuracy of two different image guidance systems in radiotherapy. Rep Pract Oncol Radiother 2016;21(3):259-65.

29. Zielińska-Dąbrowska S, Kukołowicz PF, Czebek-Szebek P. Comparison of image registration performed with MV cone beam CT and CT on rails and Syngo ${ }^{\mathrm{TM}}$ Adaptive Targeting software. Rep Pract Oncol Radiother 2009;14(4):122-32.

30. Moriya S, Tachibana H, Kitamura N, Sawant A, Sato M. Dose warping performance in deformable image registration in lung. Phys Med 2017;37:16-23.

31. Guidi G, Maffei N, Vecchi C, et al. A support vector machine tool for adaptive tomotherapy treatments: prediction of head and neck patients criticalities. Phys Med 2015;31:442-51.

32. Knopf A, Nill S, Yohannes IK, et al. Challenges of radiotherapy: report on the 4D treatment planning workshop 2013. Phys Med 2013;30:809-15.

33. Berenguer R, de la Vara V, Lopez-Honrubia V, et al. The influence of the image registration method on the adaptive radiotherapy. A proof of the principle in a selected case of prostate IMRT. Phys Med 2018;45:93-8.

34. Perna L, Sini C, Cozzarini C, et al. Deformable registration-based segmentation of the bowel on Megavoltage CT during pelvic radiotherapy. Phys Med 2016;32:898-904. 